Homoclinic Snaking near a Heteroclinic Cycle in Reversible Systems

Knobloch, J. and Wagenknecht, T.

2005

MIMS EPrint: 2006.413

Manchester Institute for Mathematical Sciences

School of Mathematics

The University of Manchester

\footnotetext{
Reports available from: http://eprints.maths.manchester.ac.uk/

And by contacting: The MIMS Secretary

School of Mathematics

The University of Manchester

Manchester, M13 9PL, UK
} 


\title{
Homoclinic Snaking near a Heteroclinic Cycle in Reversible Systems
}

\author{
J.Knobloch \\ Department of Mathematics, TU-Ilmenau, D-98684 Ilmenau, Germany \\ E-Mail: juergen.knobloch@tu-ilmenau.de \\ T. Wagenknecht ${ }^{1}$ \\ Bristol Laboratory for Advanced Dynamic Engineering, University of Bristol, \\ Queen's Building, University Walk, Bristol, BS8 1TR, UK \\ E-Mail: t.wagenknecht@bristol.ac.uk
}

\begin{abstract}
Snaking curves of homoclinic orbits have been found numerically in a number of ODE models from water wave theory and structural mechanics. Along such a curve infinitely many fold bifurcation of homoclinic orbits occur. Thereby the corresponding solutions spread out and develop more and more bumps (oscillations) about their own centre. A common feature of the examples is that the systems under consideration are reversible.

In this paper it is shown that such a homoclinic snaking can be caused by a heteroclinic cycle between two equilibria, one of which is a bi-focus. Using Lin's method a snaking of 1-homoclinic orbits is proved to occur in an unfolding of such a cycle. Further dynamical consequences are discussed.

As an application a system of Boussinesq equations is considered, where numerically a homoclinic snaking curve is detected and it is shown that the homoclinic orbits accumulate along a heteroclinic cycle between a real saddle and a bi-focus equilibrium.
\end{abstract}

Key words: bifurcation, heteroclinic cycle, homoclinic snaking, Lin's method, Boussinesq system.

PACS: 02.30.Hq, 05.45-a

$\overline{1}$ Corresponding author 


\section{Introduction}

Shilnikov's model of a homoclinic orbit to a saddle-focus equilibrium [22] is one of the most famous examples in dynamical systems theory, in which a relatively simple configuration generates a highly complicated behaviour in its neighbourhood. One of the classical results for this problem concerns the bifurcation of one-periodic orbits in a neighbourhood of the homoclinic loop. Their bifurcation can be described by the curve shown in Figure 1, see also in the textbook [14]. In this context the $\omega$ in Figure 1 denotes the minimal period of the periodic orbits and $\lambda$ is the system's parameter that unfolds the homoclinic connection. The figure shows a 'snaking' of the periodic orbits, such that for $\lambda=0$, where the homoclinic orbit exists, infinitely many periodic orbits exist. These orbits vanish in saddle-node bifurcations of periodic orbits that occur at the minima and maxima along the snaking curve. Only finitely many of these orbits survive the break-up of the homoclinic connection for $\lambda \neq 0$.

A similar snaking of homoclinic orbits has been observed more recently in a number of examples ranging from structural mechanics $[10,26]$ to water-wave theory [4] and nonlinear optics [23]. A common property of these examples is that the underlying ODE model is a reversible system. In [26] a formal discussion based on geometric arguments shows how the unfolding of a heteroclinic cycle can lead to homoclinic snaking.

In this article we will prove analytically that homoclinic snaking in reversible systems indeed occurs in perturbations of a symmetric heteroclinic cycle. The cycle thus acts as an 'organising centre' for that dynamics and can be seen as the equivalent to the Shilnikov homoclinic orbit, which 'organises' the bifurcation of periodic orbits in its neighbourhood.

In our analysis we focus on the unfolding of the heteroclinic cycle and prove, by means of Lin's method $[11,17,18]$, that homoclinic snaking occurs. More precisely we prove that in the unperturbed system the cycle is accompanied by countably infinitely many 1-homoclinic orbits (1-homoclinic with respect to the primary cycle) which accumulate at the cycle. Only finitely many of these 1-homoclinic orbits survive the break-up of the heteroclinic cycle. The bifurcation diagram for 1-homoclinic orbits is given in Figure 1; for the exact statement we refer to Theorem 1.1 below.

The general analysis is illustrated with numerical computations for one of the aforementioned examples where the snaking has been observed. The example we are interested in concerns solitary waves in a horizontal water channel, which are described by the Boussinesq equations. Numerical studies in [4] have shown that snaking of homoclinic orbits (to a symmetric real saddle) 
occurs in these equations, compare also with results in Section 3, in particular Figure 3. In Section 3 we show that actually a second equilibrium exists, which is of bi-focus type, and using the continuation software AUTO/HomCont [27] we show that there exists a heteroclinic cycle between the equilibria. This cycle is found as the limit of the snaking homoclinic orbits.

\subsection{The main result}

Let us describe the exact setting for our analysis and formulate our main theorem. We consider a one-parameter family of vector fields

$$
\dot{x}=f(x, \lambda),
$$

where $f: \mathbb{R}^{4} \times \mathbb{R} \rightarrow \mathbb{R}^{4}$ is smooth. We suppose that the family is reversible with respect to a linear involution $R$, that is

$$
R f(x, \lambda)=-f(R x, \lambda) .
$$

We refer to [25] for a collection of fundamental results about reversible systems. Observe, that as an immediate consequence of reversibility the $R$-image $R X$ of any orbit $X=\{x(t): t \in \mathbb{R}\}$ of (1) is also an orbit of the system. If $R X=X$ we call the orbit symmetric. It turns out that the orbit $X$ is symmetric if and only if its intersection with the fixed point space Fix $R:=\left\{x \in \mathbb{R}^{4}: R x=x\right\}$ of $R$ is non-empty, $X \cap$ Fix $R \neq \emptyset$. Moreover, any non-periodic symmetric orbit has exactly one intersection with Fix $R$. For the corresponding solution $x(\cdot)$ of a symmetric orbit with $x(0) \in$ Fix $R$ it holds

$$
R x(t)=x(-t) .
$$

For system (1) there should exist two symmetric hyperbolic equilibria $p_{1}$ and $p_{2}$ at $\lambda=0$. We may assume with no loss of generality that the $p_{i}$ are symmetric hyperbolic equilibria for all sufficiently small $|\lambda|$, i.e. we have $f\left(p_{i}, \lambda\right)=0$, $i=1,2$. Note further that the hyperbolicity of the equilibria implies that Fix $R$ is two-dimensional, see [25].

The eigenvalues of symmetric equilibria in reversible systems are symmetric with respect to 0 in the complex plane. As a consequence the $p_{i}$, being hyperbolic, are either real saddles (with four real eigenvalues) or bi-foci (with complex eigenvalues). The equilibrium $p_{2}$ is assumed to be a bi-focus with complex eigenvalues

$$
\sigma\left(D_{1} f\left(p_{2}, \lambda\right)\right)=\{ \pm \rho(\lambda) \pm \phi(\lambda) i\}, \quad \rho(\lambda), \phi(\lambda)>0 \text { for all } \lambda .
$$

We finally assume that a heteroclinic orbit $\Gamma_{1}=\left\{\gamma_{1}(t): t \in \mathbb{R}\right\}$ between $p_{1}$ 
and $p_{2}$ exists at $\lambda=0$. By reversibility $\Gamma_{1}$ is necessarily part of a heteroclinic cycle $\Gamma$, consisting of the two equilibria $p_{i}$ and two heteroclinic orbits $\Gamma_{1}$, $\Gamma_{2}:=R \Gamma_{1}$. In the following we impose certain non-degeneracy conditions on $\Gamma_{1}$. Again, reversibility ensures that those conditions are also fulfilled by $\Gamma_{2}$.

Throughout this paper we denote the stable manifold of $p_{i}$ with respect to the vector field $f(\cdot, \lambda)$ by $W^{s}\left(p_{i}, \lambda\right)$ and write just $W^{s}\left(p_{i}\right)$ for $W^{s}\left(p_{i}, 0\right)$. In the same manner we use $W^{u}\left(p_{i}, \lambda\right)$ and $W^{u}\left(p_{i}\right)$ to denote the corresponding unstable manifolds. By our assumptions the stable and unstable manifolds $W^{s}\left(p_{i}\right)$ and $W^{u}\left(p_{i}\right), i=1,2$ are two-dimensional. We assume the intersection of the stable and unstable manifolds along $\Gamma_{1}$ to be as clean as possible,

$$
\operatorname{dim}\left(T_{\gamma_{1}(0)} W^{u}\left(p_{1}\right) \cap T_{\gamma_{1}(0)} W^{s}\left(p_{2}\right)\right)=1 .
$$

(By $T_{q} M$ we denote the the tangent space of the manifold $M$ at $q$. )

Finally, we assume a generic unfolding of the (structurally unstable) heteroclinic connection. Let

$$
W_{\Lambda}^{s / u}:=\bigcup_{\lambda}\left(W^{s / u}\left(p_{2}, \lambda\right) \times\{\lambda\}\right) \subset \mathbb{R}^{5} .
$$

We assume that $W_{\Lambda}^{s}$ and $W_{\Lambda}^{u}$ intersect transversally at $\gamma(0):=\left(\gamma_{1}(0), 0\right)$ :

$$
W_{\Lambda}^{s} \pitchfork_{\gamma(0)} W_{\Lambda}^{u}
$$

In particular, this implies that the cycle does not exist for $\lambda \neq 0$.

With a view to the introductory remarks our goal is to determine the set of 1-homoclinic orbits to $p_{1}$. A homoclinic orbit is called 1-homoclinic (with respect to the cycle $\Gamma$ ) if it lies in a sufficiently small neighbourhood $\mathcal{U}$ of $\Gamma$ and passes a cross-section $\Sigma_{1}$ of $\Gamma_{1}$ exactly once. Our main result reads as follows:

Theorem 1.1 Consider a heteroclinic cycle $\Gamma$ as described above.

At $\lambda=0$ there exist countably infinitely many symmetric 1-homoclinic orbits to $p_{1}$. The single homoclinic orbits differ in their transition time $2 \omega$ from $\Sigma_{1}$ to $\Sigma_{2}:=R \Sigma_{1}$. The difference in transition time tends asymptotically to $\pi /(2 \phi)$.

For $\lambda \neq 0,|\lambda|$ small, there are only finitely many symmetric 1-homoclinic orbits to $p_{1}$.

With the addition of the parameter $\lambda$ to the phase space these 1-homoclinic orbits form a one-parameter family parameterised by $\omega$. The corresponding bifurcation diagram (for sufficiently large $\omega$ ) is depicted in Figure 1. 


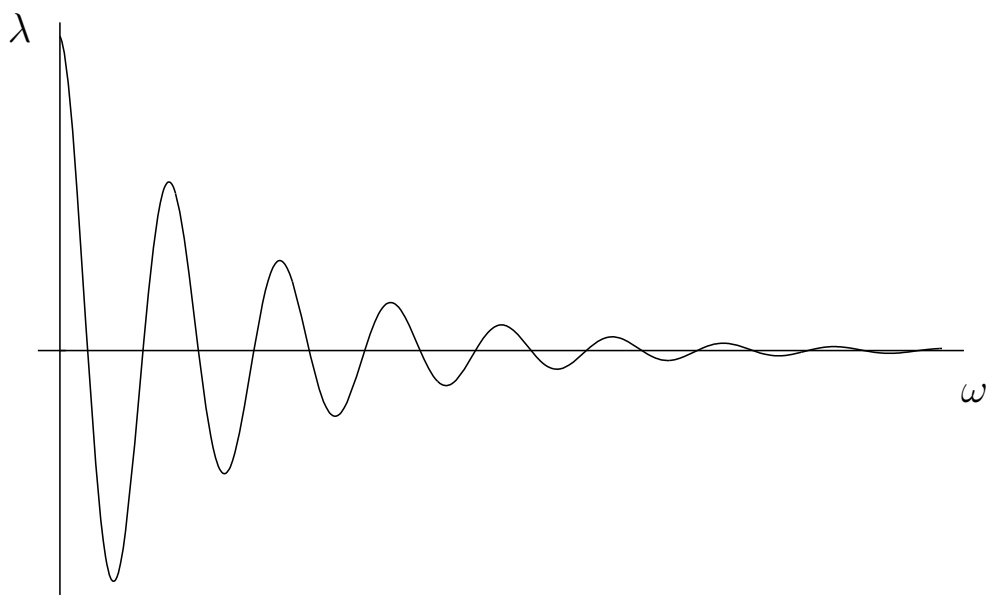

Figure 1. Bifurcation diagram for both a) one-periodic orbits bifurcating from a Shilnikov homoclinic orbit and b) 1-homoclinic orbits bifurcating from the heteroclinic cycle $\Gamma$ in Theorem 1.1. In both cases the asymptotic behaviour for large $\omega$ is depicted.

The proof of Theorem 1.1, in particular the derivation of the bifurcation equation (11), reveals more properties of the set of 1-homoclinic orbits near the cycle. First it can be shown that there are no non-symmetric 1-homoclinic orbits, see Lemma 2.2. Further, we want to emphasise that the statement of the theorem does not depend on the type (real saddle or bi-focus) of the equilibrium $p_{1}$. This can be seen from the leading term of the bifurcation equation which is determined only by the type of $p_{2}$.

Figure 1 shows that the homoclinic orbits can be continued with respect to the parameter $\lambda$. This indicates that the homoclinic orbits are robust under perturbations. Indeed, this agrees with the generic situation in reversible systems where typically the unstable manifold of the corresponding equilibrium will intersect Fix $(R)$ transversally; in our notations this reads $W^{u}\left(p_{1}\right) \pitchfork \operatorname{Fix}(R)$. This robustness will be exploited in our numerical studies.

At the minima and maxima of the bifurcation curve in Figure 1 - viewed as graph of a function $\omega \mapsto \lambda(\omega)$ - fold bifurcations of homoclinic orbits occur. There, two homoclinic orbits coalesce and vanish. This homoclinic bifurcation has been analysed in $[6,11]$. It has been shown that the bifurcation is caused by a tangency of the unstable manifold of the equilibrium and Fix $(R)$. In Section 4 we explain in a non-rigorous manner why a very complicated dynamics in a neighbourhood of the cycle may be expected. As already mentioned the emphasis in this paper is put upon the explanation of the homoclinic snaking phenomenon. But the discussion in Section 4, will give a flavour of the dynamical richness, and we will point out directions for future research. 


\subsection{Related studies}

Apart from our particular motivation the investigation of the dynamics near a heteroclinic cycle is of interest in its own right, since similar to homoclinic orbits also heteroclinic cycles can be the source for a very rich dynamics in their neighbourhood. In the example studied here this is indicated by the bifurcation of 1-homoclinic orbits near the cycle, which themselves are accompanied by complex dynamics. However, in contrast to the multitude of articles about homoclinic orbits (see for instance the overview for reversible systems in [3]), noticeably few studies of bifurcations from heteroclinic cycles in reversible or conservative systems can be found in the literature.

The existence of periodic orbits near heteroclinic cycles in reversible systems has been studied in [24]. In [13] variational methods have been used to investigate heteroclinic cycles between bi-foci in fourth-order equations that are both reversible and Hamiltonian. A bifurcation analysis of such cycles in Hamiltonian systems can be found in [16]. There, in particular, sequences of parameter values have been detected for which homoclinic fold bifurcations occur. We also refer to Section 5 in the present paper, where we give a reason for the resemblance of the sets of nearby 1-homoclinic orbits in the reversible and Hamiltonian case. But we want to note that the symbolic dynamics, which has been proved to occur near the cycle in the Hamiltonian case, see [16], cannot be expected in the reversible case; this is due the lack of level sets in the latter case. We refer to [8] for a discussion of this fact. Recent work by one of the authors concerns heteroclinic cycles near T-points in three-dimensional reversible systems, [12]; see also [15].

Finally we want to mention that several studies of generic bifurcations from heteroclinic loops in general systems, i.e. systems without any imposed structure like reversibility, have appeared in the literature, see for example [5,21]. Note that in general systems bifurcations of heteroclinic cycles are in contrast to our case at least of codimension two, since for the unfolding of each heteroclinic connection one parameter is needed. In the papers $[5,16,21]$ the bifurcation equations are derived by means of an appropriate Poincaré map.

\section{Proof of the main theorem}

We will use Lin's method to prove Theorem 1.1. Restricted to our purpose the goal of this method is to find 'piecewise continuous 1-homoclinic orbits' to $p_{1}$, which we address as 1-homoclinic Lin orbits. Such orbits consist of pieces $X_{1}^{-}, X_{12}$ and $X_{2}^{+}$of actual orbits; $X_{1}^{-}$and $X_{2}^{+}$are contained in the unstable and stable manifold, respectively, of $p_{1}$. The orbit $X_{1}^{-}$follows $\Gamma_{1}$ until it hits 
the cross section $\Sigma_{1}$, while $X_{2}^{+}$starts in $\Sigma_{2}=R \Sigma_{1}$ and follows $\Gamma_{2}$ up to the equilibrium $p_{1}$. The orbit $X_{12}$ starts in the cross-section $\Sigma_{1}$, follows $\Gamma_{1}$ until it reaches a neighbourhood of $p_{2}$ and then follows $\Gamma_{2}$ until it hits $\Sigma_{2}$ after a prescribed time $2 \omega$. (For our analysis we will assume that $\Sigma_{i}, i=1,2$ are hyperplanes intersecting $\Gamma_{i}$ at $\gamma_{i}(0)$.) Between two consecutive orbits $X_{1}^{-}$and $X_{12}$ or $X_{12}$ and $X_{2}^{+}$there may be a jump $\Xi_{1}$ or $\Xi_{2}$ in distinguished directions $Z_{1}$ or $Z_{2}=R Z_{1}$, respectively. The subspace $Z_{1} \subset T_{\gamma_{1}(0)} \Sigma_{1}$ is complementary to $T_{\gamma_{1}(0)} W^{u}\left(p_{1}\right)+T_{\gamma_{1}(0)} W^{s}\left(p_{2}\right)$. By our first transversality condition (5) we have $\operatorname{dim} Z_{1}=1$. Figure 2 depicts the described situation.

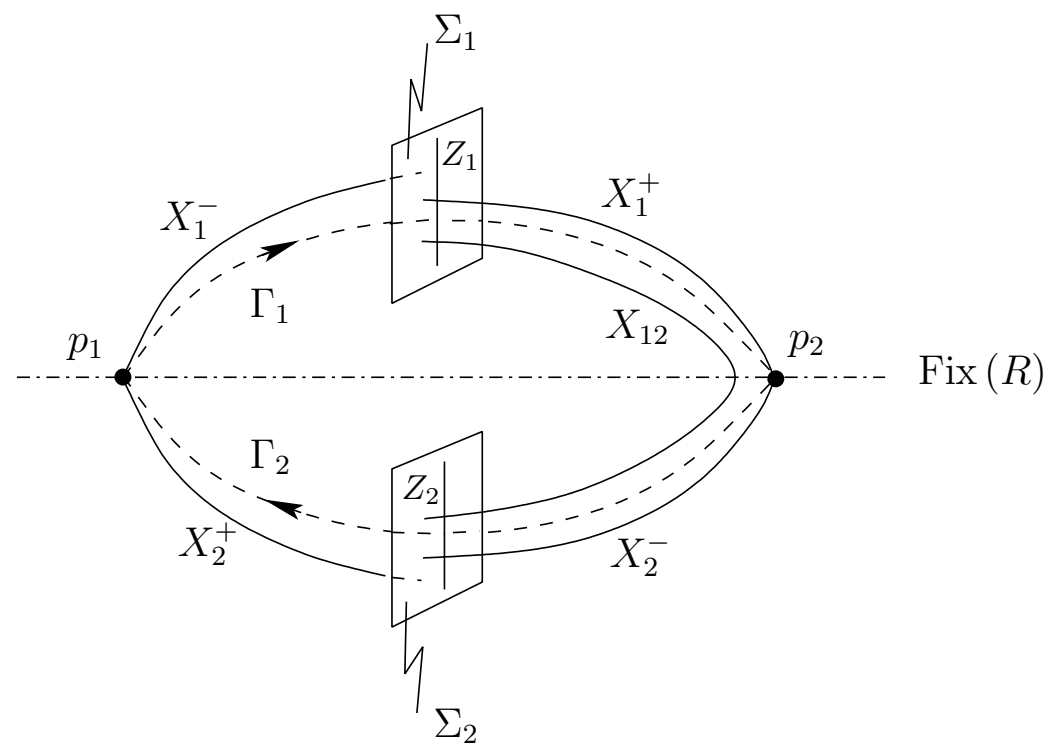

Figure 2. A 1-homoclinic Lin orbit near the heteroclinic cycle.

The first fundamental result reads.

Lemma $2.1([\mathbf{1 1}, \mathbf{1 7}, \mathbf{1 8}])$ There exist positive numbers $\hat{\lambda}, \hat{\omega}$, such that for each $\lambda,|\lambda|<\hat{\lambda}$ and each $\omega>\hat{\omega}$ there exists a unique 1-homoclinic Lin orbit $X(\omega, \lambda)$ to $p_{1}$.

Throughout the following we assume $|\lambda|<\hat{\lambda}$ and $\omega>\hat{\omega}$.

By our construction $\left(\Sigma_{2}=R \Sigma_{1}\right.$ and $\left.Z_{2}=R Z_{1}\right)$ we find that the $R$-image of $X(\omega, \lambda)$ is a 1-homoclinic Lin orbit to $p_{1}$, and what is more, $R X(\omega, \lambda)$ is related to the same parameters $\omega$ and $\lambda$. Hence, because of the uniqueness assertion in Lemma 2.1 we conclude:

Lemma 2.2 Every 1-homoclinic Lin orbit $X(\omega, \lambda)$ to $p_{1}$ is symmetric, $i$. e. $X(\omega, \lambda)=R X(\omega, \lambda)$. In particular every 1-homoclinic orbit to $p_{1}$ is symmetric. 
The Lin orbits have discontinuities only in $\Sigma_{1}$ and $\Sigma_{2}$, and therefore the detection of 1 -homoclinic orbits to $p_{1}$ amounts to solving $\Xi_{i}(\omega, \lambda)=0, i=1,2$. As a consequence of Lemma 2.2 we find $\Xi_{2}(\omega, \lambda)=R \Xi_{1}(\omega, \lambda)$. Therefore the bifurcation equation for 1 -homoclinic orbits just reads

$$
\Xi(\omega, \lambda):=\Xi_{1}(\omega, \lambda)=0 .
$$

Because $Z_{1}$ and $\lambda$ are one-dimensional $\Xi$ can be read as a mapping $\mathbb{R} \times \mathbb{R} \rightarrow \mathbb{R}$.

In $[11,18]$ expansions for the jump $\Xi$ have been derived. We apply the main results here and refer to those papers for details. The jump $\Xi(\omega, \lambda)$ can be written in the form

$$
\Xi(\omega, \lambda)=\xi^{\infty}(\lambda)+\xi(\omega, \lambda)
$$

The first term in (8) measures the splitting of $W^{u}\left(p_{1}, \lambda\right)$ and $W^{s}\left(p_{2}, \lambda\right)$ in $Z_{1}$-direction. More precisely: For each $\lambda$ close to 0 there is a unique pair $\left(x_{1}^{+}(\lambda)(\cdot), x_{1}^{-}(\lambda)(\cdot)\right)$ of solutions of $(1)$ such that

(i) $x_{1}^{ \pm}(\cdot)(0)$ smooth and $x_{1}^{ \pm}(0)(0)=\gamma_{1}(0)$;

(ii) $x_{1}^{+}(\lambda)(0) \in \Sigma_{1} \cap W^{s}\left(p_{2}, \lambda\right), \quad x_{1}^{-}(\lambda)(0) \in \Sigma_{1} \cap W^{u}\left(p_{1}, \lambda\right)$;

(iii) $\left|x_{1}^{+}(\lambda)(t)-\gamma_{1}(t)\right|$ small $\forall t \in \mathbb{R}^{+}$and $\left|x_{1}^{-}(\lambda)(t)-\gamma_{1}(t)\right|$ small $\forall t \in \mathbb{R}^{-}$;

(iv) $x_{1}^{+}(\lambda)(0)-x_{1}^{-}(\lambda)(0) \in Z_{1}$.

The solutions $x_{1}^{ \pm}$correspond to the orbits $X_{1}^{ \pm}$. With that we have

$$
\xi^{\infty}(\lambda)=x_{1}^{+}(\lambda)(0)-x_{1}^{-}(\lambda)(0) .
$$

Of course $\xi^{\infty}(0)=0$, which represents that $W^{u}\left(p_{1}\right)$ intersects $W^{s}\left(p_{2}\right)$ along $x_{1}$. Due to our second transversality condition (6) we have $D \xi^{\infty}(0) \neq 0$. So we may assume

$$
\xi^{\infty}(\lambda)=\lambda
$$

The term $\xi(\omega, \lambda)$ in (8) measures the deviation of $X_{12}$ from $W^{s}\left(p_{2}, \lambda\right)$, again in $Z_{1}$-direction. Let $x_{12}(\lambda)(\cdot)$ be the solution of (1) corresponding to $X_{12}$ such that $x_{12}(\lambda)(0) \in \Sigma_{1}$ and $x_{12}(\lambda)(2 \omega) \in \Sigma_{2}$. Then $x_{1}^{+}(\lambda)(0)-x_{12}(\lambda)(0) \in Z_{1}$, and $\xi(\omega, \lambda)$ measures this difference. Therefore $\xi(\omega, \lambda)$ will only be influenced by the behaviour of $x_{12}(\lambda)(\cdot)$ near $p_{2}$. In other words, the computation of $\xi(\omega, \lambda)$ and therefore the results about one-homoclinic orbits to $p_{1}$ do not depend on whether $p_{1}$ is a real saddle or a bi-focus.

The leading term of $\xi(\omega, \lambda)$ will be determined by the asymptotic behaviour (as $\omega \rightarrow \infty$ ) of $x_{2}^{-}(\lambda)(\omega)=R x_{1}^{+}(\lambda)(-\omega)$ and by the asymptotic behaviour of solutions of the adjoint of the variational equation along $x_{1}^{+}(\lambda)(\cdot)$ starting in $Z_{1}$. It turns out that

$$
\xi(\omega, \lambda)=e^{-2 \rho(\lambda) \omega} c(\lambda) \sin (2 \phi(\lambda) \omega+\varphi)+o\left(e^{-2 \rho(\lambda) \omega}\right),
$$


with $\rho(\lambda), \phi(\lambda)$ defined in (4). The function $c(\cdot)$ is smooth and $c(0) \neq 0$. For a detailed analysis in similar cases (where equilibria with non-real eigenvalues are involved) we refer to $[11,12,19,20]$.

We summarise the results in the final lemma.

Lemma 2.3 All 1-homoclinic orbits to $p_{1}$ near the heteroclinic cycle $\Gamma$ can be found by solving the bifurcation equation

$$
\Xi(\omega, \lambda)=\lambda+e^{-2 \rho(\lambda) \omega} c(\lambda) \sin (2 \phi(\lambda) \omega+\varphi)+o\left(e^{-2 \rho(\lambda) \omega}\right)=0 .
$$

The solution of (11) gives the bifurcation diagram in Figure 1. To see that in a more rigorous way one solves $\Xi(\omega, \lambda)=0$ for $\lambda(\omega)$ near $(\omega, \lambda)=(\infty, 0)$. Note that $\Xi(\infty, \lambda)$ is well defined and $\Xi(\infty, \lambda)=\xi^{\infty}(\lambda)=\lambda$. Moreover, a similar estimate as given in (10) for $\xi$ holds for the derivative of $\xi$ with respect to $\lambda$. This allows an Implicit Function Theorem type of argument to gain $\lambda(\omega)$.

Remark. We emphasize that the result about 1-homoclinic orbits to $p_{1}$ does not depend on whether this equilibrium is a real saddle or bi-focus. Indeed, being contained in a neighbourhood of the primary heteroclinic cycle the homoclinic orbits found in Theorem 1.1 are mainly affected by the local flow near $p_{2}$ and differ in their number of rotations around $p_{2}$ which appear as oscillations in the solution plots in Figure 4. The 1-homoclinic orbits under consideration correspond to certain intersections of the stable and unstable manifold of $p_{1}$, which we can study in $\Sigma_{1}$ : Note first that the shape of the local stable and unstable manifold of $p_{1}$ does not depend on the eigenvalues of $p_{1}$ (only the dynamics within these manifolds does). We follow these manifolds along the heteroclinic cycle until they first intersect $\Sigma_{1}$ and denote these traces of $W^{s / u}\left(p_{1}, \lambda\right)$ in $\Sigma_{1}$ by $\mathcal{T}^{s / u}(\lambda)$. While $\mathcal{T}^{u}(\lambda)$ can always be seen as a straight line, the shape of $\mathcal{T}^{s}(\lambda)$ will be influenced by the local flow near $p_{2}$. In the situation studied in Theorem 1.1 the curve $\mathcal{T}^{s}(\lambda)$ will have the form of a spiral (due to the spin near $p_{2}$ ). The analytical results now imply that for $\lambda=0$ the curves $T^{s / u}(0)$ intersect in infinitely many points in $\Sigma_{1}$, with the tip of $\mathcal{T}^{s}(0)$ located on $\mathcal{T}^{u}(0)$. For $\lambda \neq 0$ the tip of $\mathcal{T}^{s}(0)$ moves off $\mathcal{T}^{u}(0)$, and only finitely many intersections remain.

A similar geometric interpretation explains that the existence of 2-homoclinic orbits to $p_{1}$ depends crucially on the type of this equilibrium, see Section 4 where the analysis, again based on Lin's method, is carried out. 


\section{A numerical example: homoclinic snaking in a system of Bous- sinesq equations}

To illustrate the theory we deal with solitary waves in a family of Boussinesq systems of the form

$$
\begin{aligned}
& \eta_{t}+u_{x}+(u \eta)_{x}+a u_{x x x}-b \eta_{x x t}=0 \\
& u_{t}+\eta_{x}+u u_{x}+c \eta_{x x x}-d u_{x x t}=0
\end{aligned}
$$

which models waves in a horizontal water channel travelling in both directions. System (12) has been derived in [2] as a first order approximation to the full Euler equations. The function $\eta$ describes the deviation of water surface from its undisturbed position and $u$ is the horizontal velocity at a certain height above the bottom of the channel, see [2] for details.

The above family includes several well studied systems as the classical Boussinesq system for $a=b=c=0, d=1 / 3$, the Kaup system for $a=1 / 3$, $b=c=d=0$, or the regularised Boussinesq system for $a=c=0$ and $b=d=1 / 6$. We will concentrate on the last case, which has been dealt with in $[1,4]$.

Solitary waves of (12) are sought in the form $\eta(x, t)=\eta(\xi), u(x, t)=u(\xi)$, where $\xi:=x-k t$, and $k$ denotes the speed of the wave. The corresponding system of ordinary differential equations can be integrated once, and - being interested in the regularised Boussinesq system - we set $a=c=0$ and $b=1 / 6$ to obtain

$$
\begin{aligned}
& k \eta^{\prime \prime}+6 u-6 k \eta+6 u \eta=0 \\
& d k u^{\prime \prime}-k u+\eta+\frac{1}{2} u^{2}=0 .
\end{aligned}
$$

The prime here denotes differentiation with respect to $\xi$.

Solitary waves of (12) are described by homoclinic solutions to the 0-equilibrium of (13). We study such orbits numerically using the homoclinic continuation software AUTO/HomCont, [27]. In particular, we make use of the reversibility of (13). By that we mean that the corresponding first order system is reversible (see (2)) with respect to the involution

$$
R:\left(\eta, \eta^{\prime}, u, u^{\prime}\right) \mapsto\left(\eta,-\eta^{\prime}, u,-u^{\prime}\right),
$$

and investigate symmetric solutions which are in $\operatorname{Fix}(R)$ at $\xi=0$; such solutions are characterised by $\eta(\xi)=\eta(-\xi), u(\xi)=u(-\xi)$, see (3). Recall that we can expect such solutions to exist robustly. Therefore bifurcations of symmetric homoclinic orbits can be studied under variation of one parameter in (13). Moreover, symmetric homoclinic orbits can be computed by finding intersections of the unstable manifold of 0 with the fixed space Fix $(R)$, given by those 
points in phase space for which $\eta^{\prime}=u^{\prime}=0$. The corresponding algorithms are included in the HomCont package and have been used in the computations.

For $d=1 / 6$ and $k=2.5$ an explicit homoclinic solution

$$
\begin{aligned}
& \eta(\xi)=\frac{15}{4}\left(2 \operatorname{sech}^{2}\left(\frac{3}{\sqrt{10}} \xi\right)-3 \operatorname{sech}^{4}\left(\frac{3}{\sqrt{10}} \xi\right)\right) \\
& u(\xi)=\frac{15}{2} \operatorname{sech}^{2}\left(\frac{3}{\sqrt{10}} \xi\right)
\end{aligned}
$$

of (13) has been computed in [4]. Following the analysis in that paper we continue this solution under variation of $k$ until the value $k=3$ is reached and study its behaviour under variation of $d$ afterwards. The starting solution with $k=3$ is shown in panel (a) of Figure 4.

The bifurcation scenario we are interested in has been observed in [4] under variation of the parameter $d$. Increasing $d$ from the starting value $d=1 / 6$, see point (a) in Figure 3, we detect a bifurcation of the primary homoclinic solution at $d_{1}=0.2022$, where it coalesces with a second homoclinic orbit in a fold bifurcation. Continuing this second orbit with decreasing $d$ the same scenario occurs at $d_{2}=0.075$ and - after increasing $d$ - again at $d=0.15$. These $d$-values correspond the first turning points after point (a) in Figure 3.

The computations can be continued, and we find that the parameter values, at which homoclinic fold bifurcations can be observed, accumulate at some value $d_{\infty} \approx 0.1206$. In particular, the computations suggest the existence of infinitely many homoclinic solutions for $d=d_{\infty}$. An impression of this is given in Figure 3. In this diagram the $L^{2}$-norm of the homoclinic solutions is plotted against the continuation parameter $d$. It can be seen that the homoclinic solutions are distinguished by their $L^{2}$-norm, which seems to grow without bounds along the sequence of solutions. Furthermore, solutions with small $L^{2}$-norm are more robust under perturbations of $d$ than those with bigger $L^{2}$-norm.

In Figure 4 impressions of certain homoclinic solutions for parameter values along the bifurcation sequence, as indicated by (a)-(c) in Figure 3, are given. In panel (a) the $\eta$ - and $u$-component of the starting solution is shown, whereas panels (b) and (c) contain plots of homoclinic solutions with bigger $L^{2}$-norm. Along the bifurcation curve in Figure 3 the primary solution develops additional ripples around the pulse. We note that this effect has also been observed in [4] for slightly different values of $b$ and $d$.

We finally demonstrate that there exists a heteroclinic cycle between 0 and a second equilibrium of bi-focus type. By Theorem 1.1 this generically causes the snaking of the homoclinic orbits. Indeed, we see in Figure 4 that the homoclinic solution in panel (c) spends a rather long time near the values 


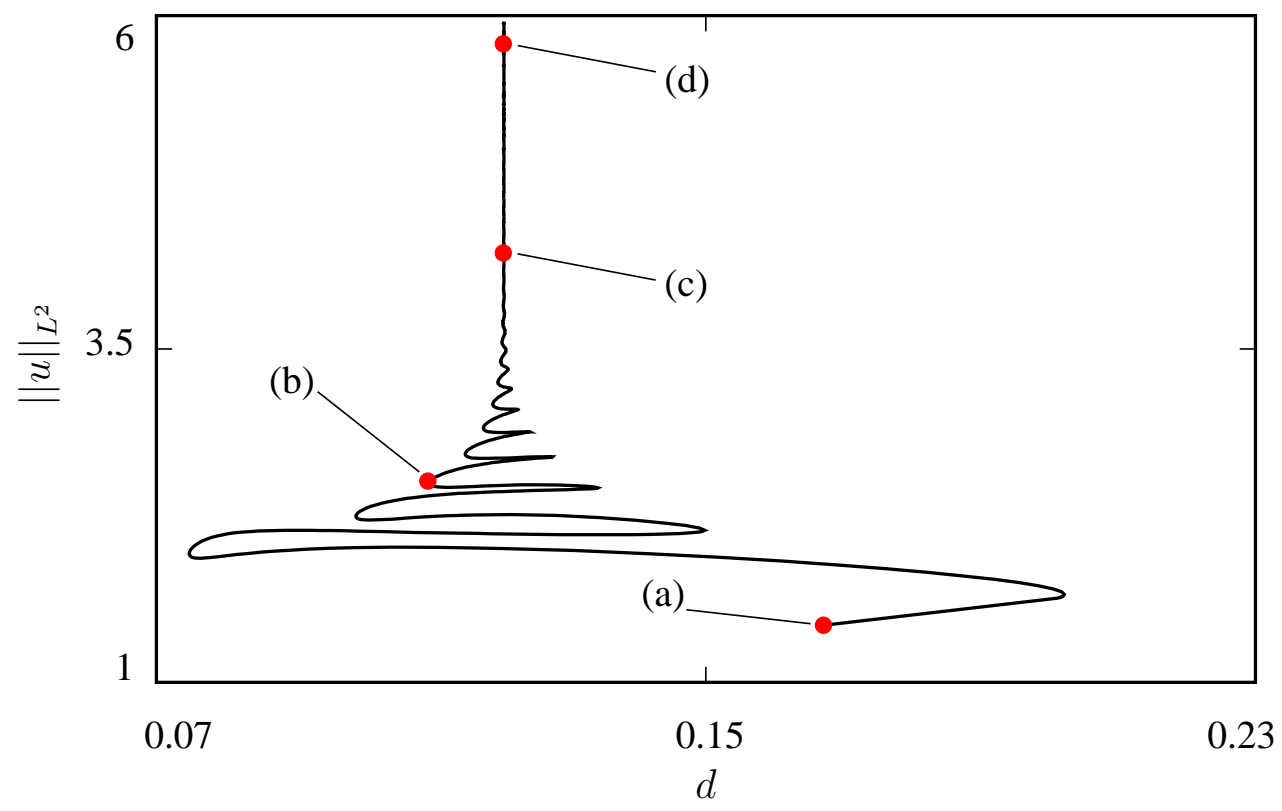

Figure 3. Bifurcation diagram for homoclinic orbits of (13) with $k=3$, derived by continuing the starting solution at point (a). Plots of the solutions at points (a)-(d) are shown in Figure 4 below.

$\hat{\eta}=-1.842$ and $\hat{u}=6.562$. A straightforward computation shows that $\hat{\eta}$ and $\hat{u}$ describe a further (symmetric) equilibrium solution of (13) for all values of $d$. Moreover, a linear analysis shows this equilibrium to be of bi-focus type, that is, its linearisation has a quadruple of complex eigenvalues.

In perfect agreement with the Theorem 1.1 the computations suggest that the homoclinic solutions along the bifurcation curve in Figure 3 accumulate at a heteroclinic cycle between the bi-focus and the 0-equilibrium. This can be verified by continuing the half part of the homoclinic solution in point (d) of Figure 3 from the 0-equilibrium to Fix $(R)$. The continuation is successful, and a plot of the derived heteroclinic solution is given in Figure 5. We note that because of the reversibility of (13) heteroclinic orbits between the equilibria will come in pairs, forming a heteroclinic cycle.

\section{More about the dynamics near the heteroclinic cycle $\Gamma$}

The homoclinic snaking causes a very complicated nearby dynamics which we will discuss in the following in more detail. Let $\Gamma_{\omega}$ be the 1-homoclinic orbit related to the transition time $\omega$ whose existence has been stated in Theorem 1.1, see also Figure 1, and let $\gamma_{\omega}(\cdot)$ be the corresponding solution with $\gamma_{\omega}(0) \in \operatorname{Fix}(R)$. If $\Gamma_{\omega}$ is robust, i.e.

$$
T_{\gamma_{\omega}(0)} W_{\lambda(\omega)}^{u}\left(p_{1}\right) \pitchfork_{\gamma_{\omega}(0)} T_{\gamma_{\omega}(0)} W_{\lambda(\omega)}^{s}\left(p_{1}\right)
$$



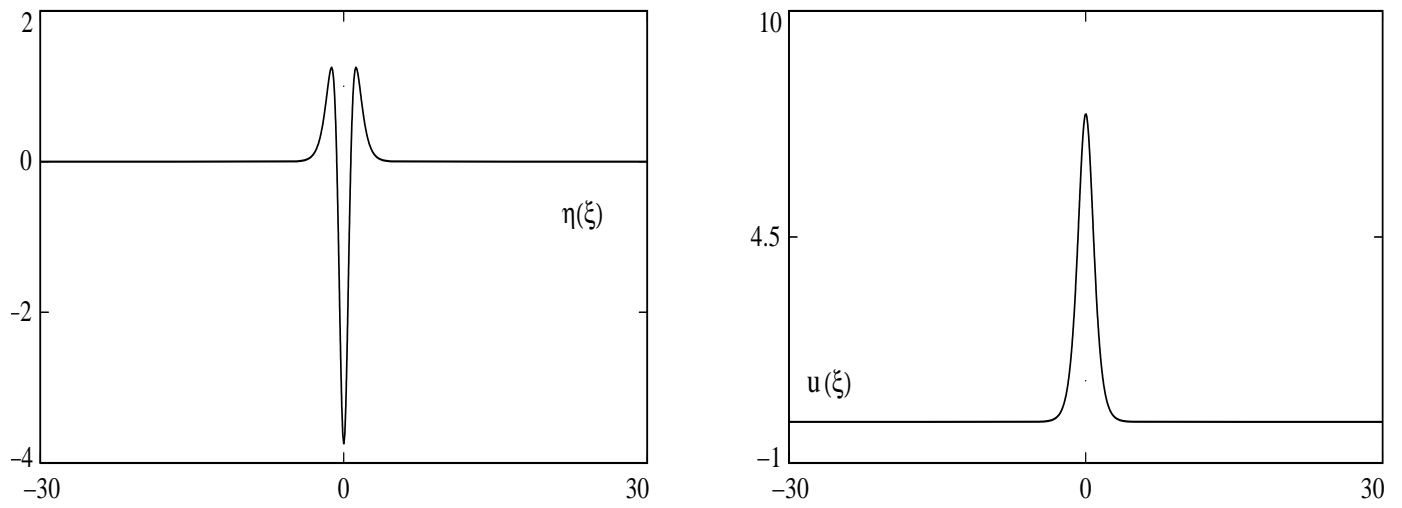

(a)
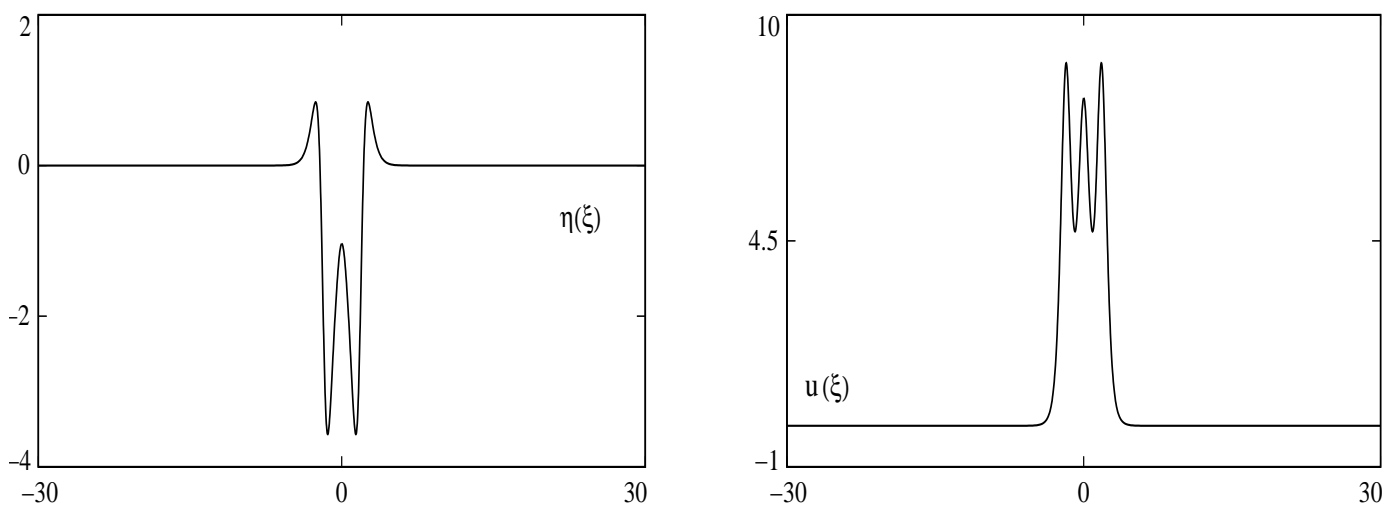

(b)
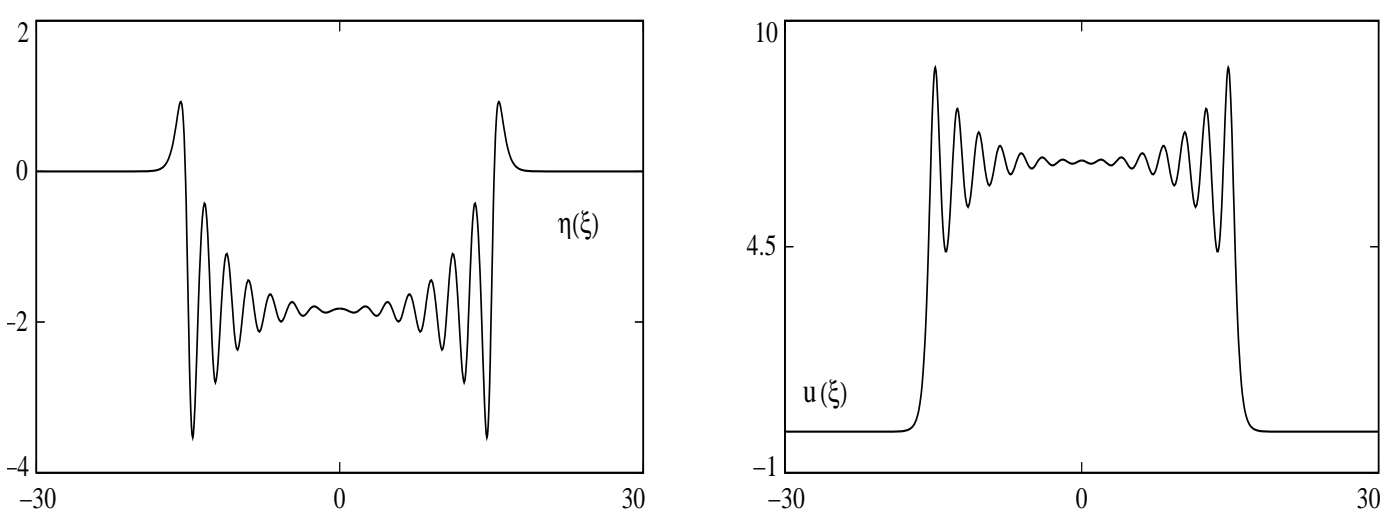

(c)

Figure 4. Homoclinic solutions of (13) for parameter values at points (a)-(c) in Figure 3 . The left parts show the $u$-component and the right parts the $\eta$-components of the solutions.

this homoclinic orbit will be accompanied by a family of symmetric 1-periodic orbits which accumulate at $\Gamma_{\omega}$, see [25].

The set of orbits that intersect $\Sigma_{1}$ or $\Sigma_{2}$ more than once, depends on the fixed point type of $p_{1}$. (Recall that the previous results are independent of this.) First we assume that $p_{1}$ is a real saddle. This is the situation we encounter in 

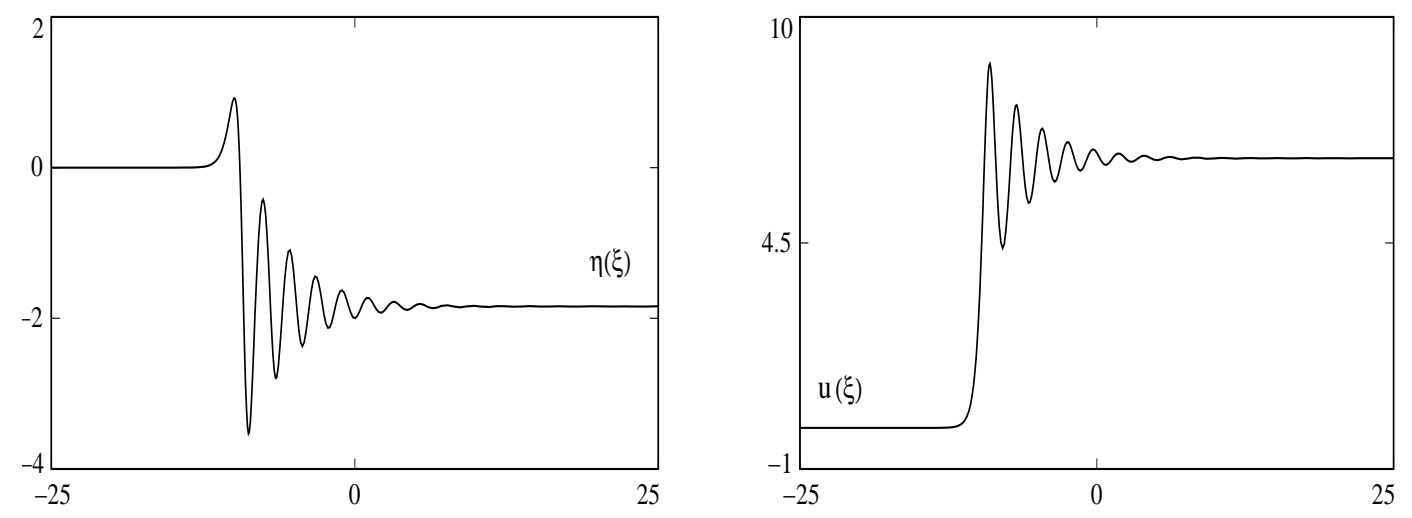

(a)

Figure 5. The heteroclinic solution between the 0-equilibrium and the equilibrium $(\hat{\eta}, 0, \hat{u}, 0)$ (in four dimensional phase space), existing for $d=d_{\infty}$.

the numerical example. In order to exclude degeneracies we will assume that $\Gamma_{1}$ approaches $p_{1}$ tangent along the leading eigendirection,

$$
\Gamma_{1} \not \subset W^{u u}\left(p_{1}\right),
$$

and we assume that the global stable manifold of $p_{2}$ intersects the local centreunstable manifold of $p_{1}$ transversally,

$$
W^{s}\left(p_{2}\right) \pitchfork_{\gamma_{1}(-T)} W_{l o c}^{c u}\left(p_{1}\right), \quad T \gg 0 .
$$

By $W^{u u}\left(p_{1}\right)$ we denote the strong unstable manifold of $p_{1}$, and by $W_{l o c}^{c u}\left(p_{1}\right)$ we denote the local centre-unstable manifold of $p_{1}$, for $\lambda=0$ in each case. The latter manifold is a flow invariant manifold whose tangent space at $p_{1}$ is spanned by the unstable and the weakest stable eigenvectors. By the reversibility similar conditions for $\gamma_{2}$ are enforced. From the homoclinic bifurcation theory (15) and (16) are known as non-orbit flip and non-inclination flip conditions, see $[18,9]$. We want to stress that our previous results do not rely on such kind of conditions.

Under these assumptions each two 1-homoclinic orbits $\Gamma_{\omega_{1}}$ and $\Gamma_{\omega_{2}}, \omega_{1} \neq \omega_{2}$, found in Theorem 1.1 form together with $p_{1}$ a reversible homoclinic bellows as considered in [8]. A bellows configuration consists of two homoclinic orbits to the same equilibrium that approach this equilibrium from the same direction for positive and similarly for negative time. (Note that under assumption (15) all detected 1-homoclinic orbits approach $p_{1}$ in the leading eigendirection, because $X_{1}^{-}$and $X_{2}^{+}$do so; this again is due to the smoothness of $x_{1}^{ \pm}(\cdot)(0)$ and the non-orbit flip condition (15).) In [8] it has been proved that for each $N \in \mathbb{N}$ there are families of symmetric $N$-periodic orbit, but there are no $N$ homoclinic orbits caused by the bellows - the $N$-periodic orbits accumulate at the bellows configuration. Here we show that there are no 2-homoclinic orbits to $p_{1}$ near the cycle; the same should be true for $N$-homoclinic orbits but the analysis is more sophisticated. 
Lemma 4.1 Let $p_{1}$ be a real saddle, and assume the non-flip conditions (15) and (16). Then there are no symmetric 2-homoclinic orbits to $p_{1}$ near $\Gamma$.

Sketch of the proof. We construct the bifurcation equation in a similar way as in Section 2. For that we consider 2-homoclinic Lin orbits; those orbits consist of pieces $X_{1}^{-}, X_{12}^{i}, X_{21}, X_{2}^{+}, i=1,2$, of actual orbits. In accordance with our previous notations $X_{12}^{i}$ connect $\Sigma_{1}$ and $\Sigma_{2}$ and $X_{21}$ connects $\Sigma_{2}$ and $\Sigma_{1}$ in forward time in each case. The upper index $i$ counts the revolutions along $\Gamma$. The corresponding jumps $\Xi_{k}^{i}$ are parallel to $Z_{k}, i, k=1,2$; Figure 6 depicts the described situation.

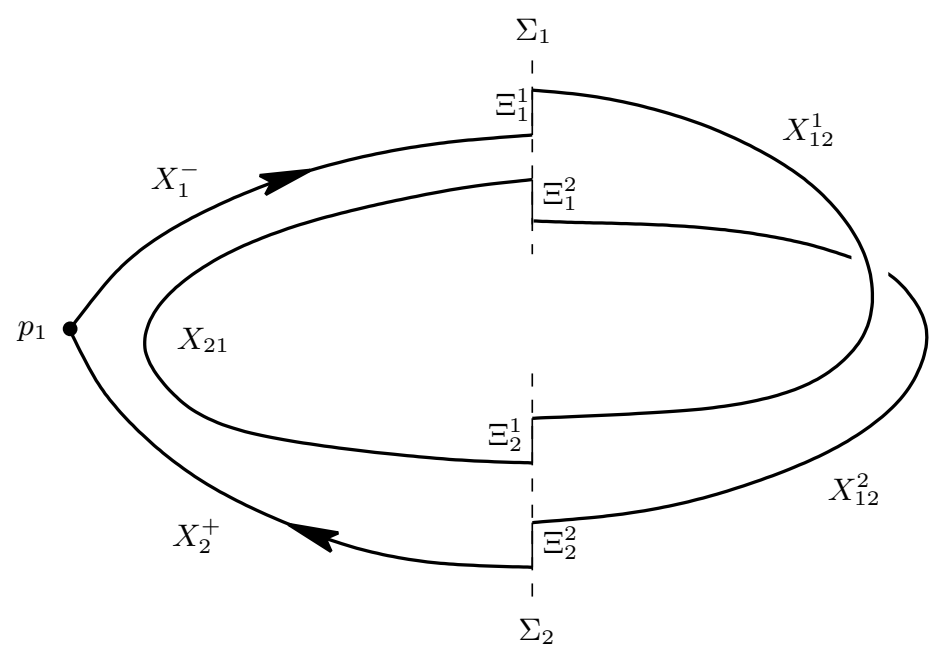

Figure 6. A 2-homoclinic Lin orbit near the heteroclinic cycle.

A 2-homoclinic Lin orbit is symmetric if

$$
R X_{12}^{1}=X_{12}^{2}, \quad R X_{1}^{-}=X_{2}^{+}
$$

This implies

$$
R \Xi_{1}^{1}=\Xi_{2}^{2}, \quad R \Xi_{1}^{2}=\Xi_{2}^{1} .
$$

Tracing further the procedure of Section 2 we end up with the bifurcation equation for symmetric 2 -homoclinic orbits to $p_{1}$ :

$$
\begin{array}{ll}
\lambda+e^{-2 \rho \omega_{12}} c \sin \left(2 \phi \omega_{12}+\varphi\right)+o\left(e^{-2 \rho \omega_{12}}\right) & =0 \\
\lambda+d e^{-2 \mu \omega_{21}}+e^{-2 \rho \omega_{12}} c \sin \left(2 \phi \omega_{12}+\varphi\right)+o\left(e^{-2 \rho \omega_{12}}\right)+o\left(e^{-2 \mu \omega_{21}}\right) & =0
\end{array}
$$

Here $\omega_{i j}$ denotes the transition time from $\Sigma_{i}$ to $\Sigma_{j}$; due to the required symmetry of the 2-homoclinic orbits $X_{12}^{1}$ and $X_{12}^{2}$ take the same time. Further $\mu$ denotes the principal unstable eigenvalue of $p_{1}$. Note that the quantities $c, d$ $\rho, \mu$ and $\phi$ depend on $\lambda$, and for the smooth function $d(\cdot)$ it holds $d(0) \neq 0$. 
By means of a procedure taking its pattern from the proof of the implicit function theorem this system can be uniquely solved for $\left(\lambda, \omega_{21}\right)\left(\omega_{12}\right)$ near $\left(\lambda, \omega_{12}, \omega_{21}\right)=(0, \infty, \infty)$. Note that the transition time " $\infty$ " corresponds to a pair of partial orbits in the stable and unstable manifolds like $\left(X_{2}^{+}, X_{1}^{-}\right)$ or $\left(X_{1}^{+}, X_{2}^{-}\right)$, see Figure 2. Let $(\lambda(\omega), \omega)$ be the solution of the bifurcation equation (11) for 1 -homoclinic orbits to $p_{1}$. Then $\left(\lambda\left(\omega_{12}\right), \omega_{12}, \infty\right)$ solves the above bifurcation equation (17) for 2-homoclinic orbits; for $\omega_{21}=\infty$ both equations in (17) coincide, and moreover they coincide with Equation (11). So, due to the uniqueness, we find $\omega_{21}\left(\omega_{12}\right) \equiv \infty$.

If $p_{1}$ is a bi-focus then we face a much more involved dynamics. In this case each 1 -homoclinic orbit to $p_{1}$ is accompanied by infinitely many $N$-homoclinic orbits for each $N \in \mathbb{N}$, see [7]. Again, all these orbits form homoclinic bellows such that an abundance of complexity can be found near the cycle.

In the same way as we searched for 1 -homoclinic orbits to $p_{1}$ we can do that for 1-homoclinic orbits to $p_{2}$. If $p_{1}$ is a bi-focus the bifurcation equation for those orbits coincides with (11). If $p_{1}$ is a real saddle the bifurcation equation reads

$$
\lambda+d(\lambda) e^{-2 \mu(\lambda) \omega}+o\left(e^{-2 \mu(\lambda) \omega}\right)=0,
$$

where $\omega$ is the transition time from $\Sigma_{2}$ to $\Sigma_{1}$. If non-flip conditions are fulfilled then $d(0) \neq 0$. Therefore either for $\lambda>0$ or for $\lambda<0$ there exists a unique 1-homoclinic orbit to $p_{2}$. We remark that in either case the results in [7] imply that a complicated set of $N$-homoclinic orbits exists whenever a 1-homoclinic to the saddle-focus $p_{2}$ exists.

\section{Discussion}

In this paper we have discussed the unfolding of a heteroclinic cycle between two equilibria in a reversible system. It has been shown that generically homoclinic snaking occurs if one of the two equilibria is of saddle-focus type. The bifurcation results have been illustrated by numerical investigations for a system of Boussinesq equations. We have also discussed possible consequences for the recurrent dynamics near the cycle.

Our approach allows an immediate generalisation to higher dimensions. Independently of the dimension of the phase space we get a single one-dimensional bifurcation equation for 1-homoclinic orbits. Instead of (4) we simply assume that the leading eigenvalues of $p_{2}$ are complex. Under conditions similar to (15) and (16) the structure of the bifurcation equation (11) will be preserved, and, finally, the reversibility of the system yields a reduction of the bifurcation equation to a single equation, see the consequences of Lemma 2.2. 
In a similar way one can treat the bifurcation of the heteroclinic cycle in the class of conservative systems, that is, systems possessing a first integral. To be more precise, let us assume that (1) has a smooth first integral $H$, i.e. $H: \mathbb{R}^{4} \times$ $\mathbb{R} \rightarrow \mathbb{R}$ is smooth and constant along orbits of (1). Moreover we will assume that $D_{1} H$ is non-singular along $\Gamma_{1} \cup \Gamma_{2}$. In this case, without reversibility, the existence of a heteroclinic orbit $\Gamma_{1}$ does not imply the existence of a second one, $\Gamma_{2}$. Hence, we have to assume the existence of a cycle. Although generically the stable and unstable manifolds of the equilibria will intersect transversally within a level set of $H$, one parameter is still needed for the unfolding of the cycle. By changing that parameter the involved equilibria will move to different level sets, and hence the cycle will break up.

In order to show that results similar to Theorem 1.1 are also valid in the conservative case we have to investigate whether a similar reduction to a onedimensional bifurcation equation is possible. The next lemma asserts exactly this.

Lemma 5.1 If $\Xi_{1}(\omega, \lambda)=0$ for some $(\omega, \lambda)$, then also $\Xi_{2}(\omega, \lambda)=0$.

As a consequence of this lemma it remains to consider only the single bifurcation equation $\Xi(\omega, \lambda):=\Xi_{1}(\omega, \lambda)=0$.

Sketch of the proof. By our assumptions both $Z_{1}$ and $Z_{2}$ are transversal to the level sets of $H(\cdot, \lambda)$ which we may assume to be flat in a neighbourhood of $\gamma_{1}(0)$ and $\gamma_{2}(0)$.

Let $\Xi_{1}=0$. Then $X_{1}^{-} \cup X_{12}$ forms a partial orbit lying in the unstable manifold of $p_{1}$. Hence $X_{1}^{-} \cup X_{12}$ and $X_{2}^{+}$are in the same level set of $H$, and therefore $\Xi_{2}=0$.

Consequently, we can derive a bifurcation equation for 1-homoclinic orbits to $p_{1}$ in the same way as in Section 2. Based on our discussion in Section 4 we can expect a very complex dynamical behaviour near the cycle $\Gamma$. It is worth mentioning that bellows to real saddles ( $p_{1}$ real saddle) in conservative systems (in difference to reversible systems) cause families (parameterised by the level sets) of shift dynamics, see [8]. If $p_{1}$ is a bi-focus one can expect an even more involved dynamics. In [16] a description of that dynamics is presented for the case that $f$ is a Hamiltonian vector field.

We finally remark that in [26] homoclinic snaking near a heteroclinic cycle between a bi-focus and a periodic orbit has been discussed. Such a cycle emerges in a supercritical Hamiltonian-Hopf bifurcation or reversible 1:1 resonance. In this case the amplitude of the 'horizontal snaking' does not decay to zero along the curve, but the horizontal minima and maxima approach certain values $\lambda_{-}$ and $\lambda_{+}$, see for instance Figure 14 in [26]. A formal argument in that paper 
shows that $\lambda_{-}$and $\lambda_{+}$are related to the range of existence of the heteroclinic cycle, which is structurally stable. We expect that similar snaking curves arise near structurally stable cycles between equilibria, for instance in systems with a reversibility that maps the equilibria onto each other. (Note that in this case both equilibria are bi-foci.) A detailed analysis is left for future work.

Acknowledgments. The authors thank Alan Champneys for his explanations of the 'snaking problem'. T.W.'s research has been supported by the EPSRC grant number GR/535684/01.

\section{References}

[1] J. L. Bona and M. Chen. A Boussinesq system for two-way propagation of nonlinear dispersive waves. Physica D, 116:191-224, 1998.

[2] J. L. Bona, M. Chen, and J.-C. Saut. Boussinesq equations and other systems for small amplitude long waves in nonlinear dispersive media I: Derivation and the linear theory. J. Nonlinear Sci., 12:283-318, 2002.

[3] A. R. Champneys. Homoclinic orbits in reversible systems and their applications in mechanics, fluids and optics. Physica D, 112(1-2):158-186, 1998.

[4] M. Chen. Solitary-wave and multi-pulsed travelling-wave solutions of Boussinesq systems. Appl. Anal., 75(1-2):213-240, 2000.

[5] Chow, S.-N., Deng, B. and Terman, D., The bifurcation of homoclinic and periodic orbits from two heteroclinic orbits, SIAM J. Math. Anal. 21(1):179$204,1990$.

[6] B. Fiedler and D. Turaev. Coalescence of reversible homoclinic orbits causes elliptic resonance. Int. J. Bifurcation Chaos 6:1007-1027, 1996.

[7] J. Härterich. Cascades of reversible homoclinic orbits to a bi-focus equilibrium. Physica D, 112:187-200, 1998.

[8] A. J. Homburg and J. Knobloch. Multiple homoclinic orbits in conservative and reversible systems. Trans. Amer. Math. Soc., to appear.

[9] Homburg, A.J. and Krauskopf, B., Resonant homoclinic flip bifurcation. J. Dyn. Differential Equations, 12:807-850, 2000.

[10] G. W. Hunt, M. A. Peletier, A. R. Champneys, P. D. Woods, M. Ahmer Wadee, C. J. Budd and G. J. Lord. Cellular buckling in long structures. Nonlinear Dynamics, 21:3-29, 2000.

[11] J. Knobloch. Lin's method for discrete and continuous dynamical systems and applications. TU Ilmenau 2004. http://imath.mathematik.tu-ilmenau.de/ knobi

[12] J. Knobloch, J. S. W. Lamb and K. Webster, Shift dynamics in a neighborhood of a T-point containing two saddle-foci. In preparation. 
[13] W. D. Kalies, J. Kwapisz and R. C. A. M. VanderVorst. Homotopy Classes for Stable Connections between Hamiltonian Saddle-Focus Equilibria. Comm. Math. Pysics, 193(2):337-371, 1998.

[14] Y. Kuznetsov. Elements of Applied Bifurcation Theory. Springer Verlag 1998.

[15] J. S. W. Lamb, M. A. Teixeira and K. Webster. Heteroclinic cycle bifurcations near Hopf-zero bifurcation in reversible vector fields in $\mathbb{R}^{3}$. J. Differential Equations, in press, 2004.

[16] L. M. Lerman. Homo- and heteroclinic orbits, hyperbolic subsets in a oneparameter unfolding of a Hamiltonian system with heteroclinic contour with two saddle-foci. Regular and Chaotic Dynamics, 2(3-4):139-155, 1997.

[17] X. B. Lin. Using Melnikovs method to solve Shilnikovs problems. Proc. Roy. Soc. Edinburgh, 116A:295-325, 1990.

[18] B. Sandstede. Verzweigungstheorie homokliner Verdopplungen. IAAS Report No. 7, 1993.

[19] B. Sandstede. Instability of localized buckling modes in a one-dimensional strut model. Phil. Trans. R. Soc. London, A355:2083-2097, 1998.

[20] B. Sandstede. Stability of multi-pulse solutions. Trans. Amer. Math. Soc., 350(2):429-472, 1998.

[21] Shashkov, M. V. and Turaev, D. V. On the complex Bifurcation set for a system with simple dynamics Int. J. Bif. Chaos, 6(5):949-968, 1996.

[22] L. P. Shilnikov. A case of the existence of a countable number of periodic motions. Sov. Math. Dokl., 6:163-166, 1965.

[23] D. V. Skryabin and A. R. Champneys. Walking cavity solitons. Phys. Rev. E, 63:066610, 2001.

[24] A. Vanderbauwhede. Heteroclinic cycles and periodic orbits in reversible systems. In Ordinary and delay differential equations, Pitman Res. Notes Math. Ser. 272:250-253, 1992.

[25] A. Vanderbauwhede and B. Fiedler. Homoclinic period blow-up in reversible and conservative systems. Z. Angew. Math. Phys., 43:292-318, 1992.

[26] P. D. Woods and A. R. Champneys. Heteroclinic tangles and homoclinic snaking in the unfolding of a degenerate reversible Hamiltonian-Hopf bifurcation. Physica $D, 129: 147-170,1999$.

[27] E. J. Doedel, A. R. Champneys, T. R. Fairgrieve, Yu. A. Kuznetsov, B. Sandstede, and X. J. Wang. AUTO97 continuation and bifurcation software for ordinary differential equations, 1997. Available by anonymous ftp from FTP.CS.CONCORDIA.CA, directory PUB/DOEDEL/AUTO. 